he had been after the myocardial infarction in March 1967. Thereafter he was maintained on lignocaine infusion $1 \mathrm{mg} . / \mathrm{min}$. to a maximum of $500 \mathrm{mg}$. in 24 hours and remained in sinus rhythm for 48 hours with only occasional extrasystoles. By this time the infusion had been discontinued and he felt well, was in sinus rhythm 80/min., and the blood pressure was 120/70.

At this time he suddenly relapsed to the original dysrhythmiasupraventricular tachycardia with right bundle-branch block. On this occasion, as an alternative to further electrical cardioversion but while being monitored on the "cardioverter," he was given a sharp blow on the sternum with no effect. A similar heavy thump with the ulnar side of the clenched fist on the precordium at the cardiac apex immediately induced sinus rhythm with left bundlebranch block (see Fig.). Over the next few days he had runs of ventricular extrasystoles, bigeminy, and multifocal extrasystoles requiring further lignocaine infusion followed by tabs. phenytoin $100 \mathrm{mg}$. t.i.d.

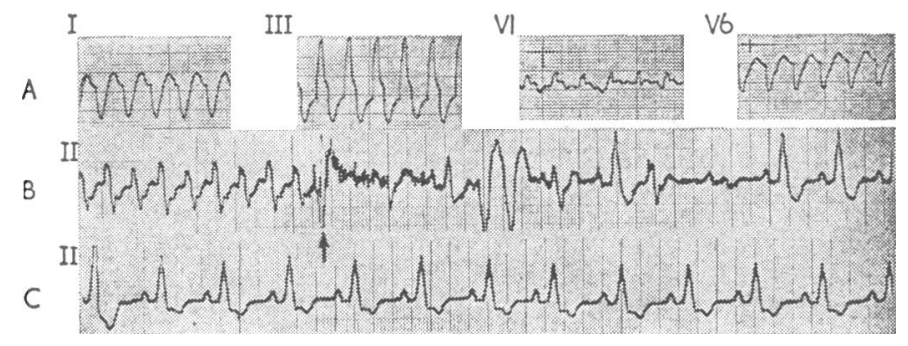

A, Tracings showing supraventricular tachycardia with right bundlebranch block in a selection of leads. B, Effect of a precordial blow ( $\uparrow$ ) showing sinus beats, ventricular extrasystoles, and eventually left bundlebranch biock. C, Stabilization in left bundle-branch block a few seconds

One week later, while he was still being monitored, his rhythm again relapsed to supraventricular tachycardia with right bundlebranch block, and once more sinus rhythm was easily induced by a single precordial blow. A tendency to recurring ventricular extrasystoles persisted, however, and the phenytoin dose was increased to $400 \mathrm{mg}$. daily.

On 7 August, three weeks after the original electrical cardioversion, he again developed tachycardia. He was no longer being monitored, but an E.C.G. tracing showed supraventricular tachycardia, this time with left bundle-branch block. Once more a precordial thump induced sinus rhythm and left bundle-branch block with multifocal extrasystoles, which settled on administration of antiarrhythmic drugs lignocaine and phenytoin. He had no further episodes of arrhythmia, his E.C.G. showing the same picture of left bundle-branch block as it had done after the initial myocardial infarction in March 1967.

By 21 August he was getting up in the ward. Clinically and radiologically his heart was enlarged, but, on daily diuretic, there was no evidence of failure. He was still having fairly frequent ventricular extrasystoles, but was well enough to be discharged from hospital on 30 August, continuing on phenytoin $100 \mathrm{mg}$. t.i.d. as an antiarrhythmic drug.

\section{Haemodynamic Effects of Balloon Septostomy in Tricuspid Atresia}

Brit. med. F., 1968, 1, 225-226

Tricuspid atresia has been regarded as one of the rare congenital cardiac anomalies, but it must be considered in the differential diagnosis of every infant with cyanotic heart disease. Campbell (1955) stated that tricuspid atresia is present in about $5 \%$ of patients with cyanotic congenital heart disease ; Keith et al. (1958) placed the incidence at $3.2 \%$.

The physiological consequences of the anomaly are manifold. Arterial desaturation is caused by complete mixing of systemic venous and pulmonary venous blood in the left atrium. The jegree of unsaturation and cardiac handicap depends on the
He was readmitted on 6 September again in supraventricular tachycardia and with evidence of congestive failure. On three occasions over the next 48 hours a precordial thump converted this rhythm to sinus rhythm. On each occasion the procedure was performed with the patient monitored on the cardioverter, and with the shock paddles prepared and an intravenous drip available for giving sodium bicarbonate or other drugs in the event of more serious arrhythmia developing. In view of the continuing cardiac irritability with multifocal extrasystoles he was then put on a procainamide continuous infusion, $4 \mathrm{~g}$. in 24 hours, which had the effect of almost clearing the ventricular ectopic beats.

\section{COMMENT}

Thus physical stimulation of the heart, like electrical stimulation and drugs such as digitalis and quinidine, would seem to have the ability to induce and reverse arrhythmias. In our patient it seems that the direct-current precordial shocks given during the first attack of arrhythmia were too powerful. It will be interesting to discover whether this simple physical method can be used as an alternative to electrical cardioversion in other types of arrhythmia. It has the advantage that no anaesthetic is necessary, but we would strongly advise that the patient's E.C.G. be monitored, with a defibrillator to hand, when the stimulus is given. There is evidence that such a blow can produce an ectopic beat which, by falling in the vulnerable $\mathrm{T}$-wave zone, might conceivably induce ventricular fibrillation (Smirk and Palmer, 1960). This could account for the very rare and otherwise inexplicable sudden death to the boxing ring.

It seems that response to electrical and physical stimulation is intensified in the diseased and hypoxic myocardium and by certain drugs such as digitalis (Scherf and Bornemann, 1960), and a single blow, instead of eliciting a single ectopic beat as it does in the normal heart, might well induce unwelcome tachycardia or ventricular fibrillation in the presence of such cardiac instability.

Unfortunately there is no way of synchronizing the stimulus. Thus until more is known about the possible dangers of this treatment its use should be restricted to cardiologists with experience in treating acute arrhythmias, employing all the emergency precautions normally taken with planned electrical precordial shock.

T. SEMPLE, M.D., B.SC., F.R.C.P.ED., F.R.C.P.GLAS., M.R.C,P, R. H. AL BADRAN, M.B., CH.B.

B. E. BOYES, M.B., CH.B.

Cardiac Department, Victoria Infirmary, Glasgow S.2.

\section{REFERENCBS}

Don Michael, T. A., and Stanford, R. L. (1963). Lancet, 1, 699. Scherf, D., and Bornemann, C. (1960). Amer. \}. Cardiol., 5, 30. Semple, T., Dall, J. L. C., Lancaster, W. M., Wang, I., Grigor, K., and Peel, A. A. F. (1963). Scot. med. 7., 8, 475. Smirk, F. H., and Palmer, D. G. (1960). Amer. F. Cardiol., 6, 620.

pulmonary flow and the size of the atrial septal opening. If the atrial septal defect is small it causes raised right atrial pressure, and this in turn results in raised systemic venous pressure and severe congestive failure. In such cases enlargement of the atrial septal defect should decrease central venous pressure and thus relieve the congestive heart failure. We report here the application of the technique of atrial septostomy in a small infant with tricuspid atresia and transposition of the great vessels with an extremely small intra-atrial communication.

\section{CASE RePort}

A full-term male infant born on 9 February 1967 and weighing $7 \mathrm{lb} .6 \mathrm{oz}$. $(3,350 \mathrm{~g}$.) was noted to be slightly cyanosed at birth. For two weeks, while the infant was nursed at home, he became dyspnoeic and more cyanosed after feeds. He was admitted to 
the Children's Hospital, Birmingham, on 22 February. Physical examination showed severe central and peripheral cyanosis, rapid respiration, enlargement of the liver, and a regular pulse rate of $160-180 /$ minute. The heart was clinically enlarged and there was a loud grade II systolic murmur in the third left intercostal space. Chest radiography revealed a slightly enlarged heart, tending to be "square" in shape as in tricuspid atresia. The vascularity of the lung was possibly slightly increased. The E.C.G. showed left axis deviation with tall $(4 \mathrm{~mm}$.) peaked $\mathrm{P}$ waves in limb leads II and III, indicating right atrial strain.

A venous cine-angiocardiogram (23 February) from the right saphenous vein showed that the contrast medium passed from the right to the left atrium through a small opening, then to the left ventricle and great vessels. There was no right ventricular filling from the right atrium.

The aorta arose anteriorly, and a slightly enlarged pulmonary artery lay behind it in transposed position. This confirmed the diagnosis of tricuspid atresia, with, in addition, transposition of the great vessels. Digoxin and diuretics did not improve the congestive heart failure. Cardiac catheterization was undertaken on 28 February. Right atrial pressure was high, with tall $\mathrm{P}$ waves measuring 10-12 $\mathrm{mm}$. The left atrial pressure was low, the mean pressure being $0-1 \mathrm{~mm}$. $\mathrm{Hg}$.

The right femoral vein was then exposed, and at the junction of the saphenous vein a size 6 Fogarty embolectomy balloon catheter was introduced, opacified by the injection of contrast medium into its lumen. The catheter entered the right atrium, and its tip was manipulated through a small intra-atrial opening into the left atrium. The balloon was inflated with $2 \mathrm{ml}$. of contrast medium (Fig. 1) and was jerked back into the right atrium. The balloon was then deflated.

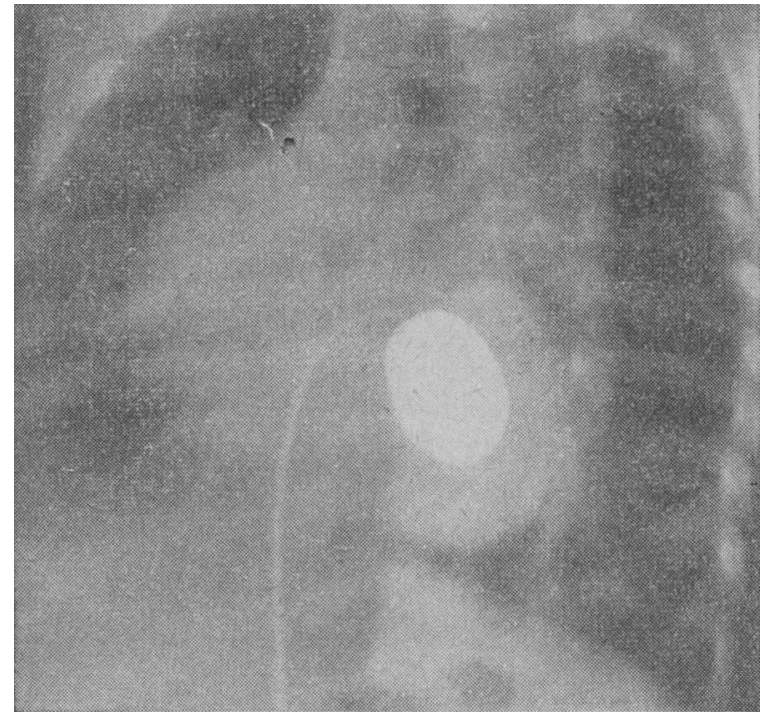

FIG. 1.-Embolectomy catheter with balloon inflated in left atrium.

The procedure was repeated with $2.5 \mathrm{ml}$. and $3.5 \mathrm{ml}$. of contrast in the balloon. The septostomy catheter was then replaced by an ordinary size 6 catheter for pressure measurements. The pressures in the right and left atrium were identical, and there was now no gradient across the interatrial opening. Unfortunately, during the last pull of the balloon catheter the patient developed supraventricular tachycardia with a ventricular rate of $220 / \mathrm{min}$. Treatment, including D.C. defibrillation, had no effect on his arrhythmia ; he deteriorated and died the next day.

Necropsy confirmed the clinical diagnosis. There were two defects in the interatrial septum, with ragged edges, separated by a thick membraneous structure $0.3 \mathrm{~cm}$. in width. The appearances suggested that the operative mancuvres had produced adequate interatrial communication (Fig. 2).

\section{COMment}

The technique for creating or enlarging an atrial septal defect is the same as that described by Rashkind and Miller (1966), and as first practised by us in cadavers in 1965 and later used in 29 cases of transposition of great vessels (November 1966 to December 1967). In cadavers we failed to obtain a satisfactory tear. This may have been due to unsatisfactory selection of material for our more recent experience with balloon septostomy for the treatment of transposition of great vessels suggests that good results are more likely to be obtained in neonates. By the age of 3 to 4 months the septum resists tearing and the foramen ovale closes when the catheter is withdrawn.

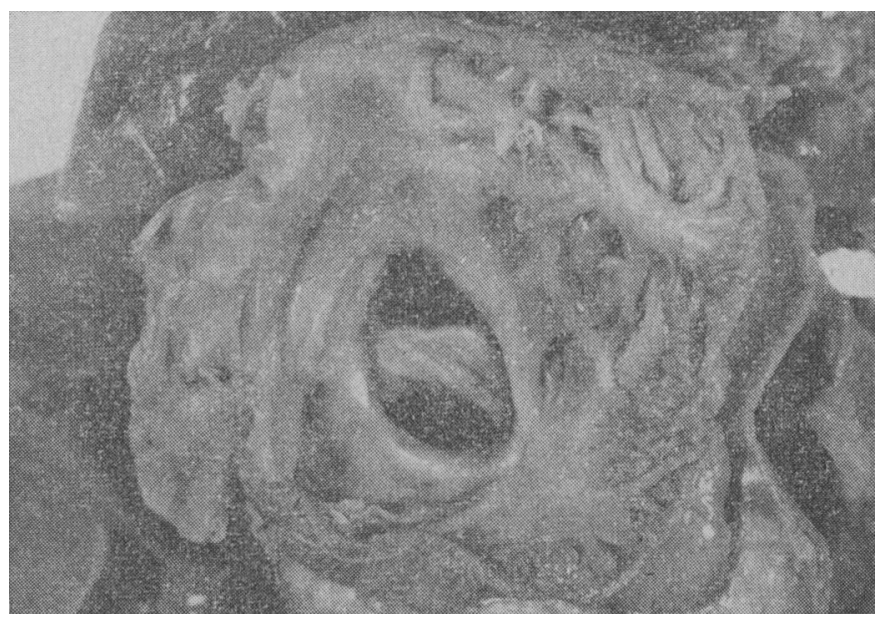

FIG. 2.-Necropsy specimen with two defects in interatrial septum created by septostomy.

We report this particular case history because although balloon septostomy is coming into widespread use (Watson and Rashkind, 1967 ; Jordan and McCarthy, 1967) for treatment of transposition, it has not yet been widely applied in tricuspid atresia. In addition it may be useful to know that such procedures can be carried out with an embolectomy catheter, an instrument that is more readily available and in greater range of sizes than the Rashkind balloon catheter.

The method has only a restricted use in tricuspid atresia, but in infants with a small interatrial communication a satisfactory opening can be obtained and the pressure gradient between the atria can be abolished.

S. P. SINGH, M.B., B.S., M.R.C.P.ED., Consultant Cardiologist, United Birmingham Hospitals and Birmingham Regional Hospital Board.

R. ASTLEY, M.D., D.M.R.,

Consultant Radiologist, Birmingham Children's Hospital

C. G. PARSONS, M.D., F.R.C.P.,

Consultant Cardiologist, United Birmingham Hospitals.

\section{REFERENCES}

Campbell, M. (1955). Brit. med. Bull., 11, 178.

Jordan, S. C., and McCarthy, C. (1967). Lancet, 1, 310.

Keith, J. D., Rowe, R. D., and Vlad, P. (1958). Heart Disease in Infancy and Childhood. New York.

Rashkind, W. J., and Miller, W. W. (1966). F. Amer. med. Ass., 196. 991.

Watson, H., and Rashkind, W. J. (1967). Lancet, 1, 403. 